\title{
Le couple générationnel dans le théâtre jeunesse : une dualité peut en cacher une autre
}

Generational Couples in Theatre for Young People: When a Duality Hides

Another One

Marie Bernanoce

\section{OpenEdition}

Journals

Édition électronique

URL : https://journals.openedition.org/recherchestravaux/739

DOI : 10.4000/recherchestravaux.739

ISSN : 1969-6434

Éditeur

UGA Éditions/Université Grenoble Alpes

Édition imprimée

Date de publication : 28 mai 2015

Pagination : 83-92

ISBN : 978-2-84310-298-1

ISSN : 0151-1874

\section{Référence électronique}

Marie Bernanoce, «Le couple générationne/ dans le théâtre jeunesse : une dualité peut en cacher une autre », Recherches \& Travaux [En ligne], 86 | 2015, mis en ligne le 28 novembre 2016, consulté le 29 octobre 2021. URL : http://journals.openedition.org/recherchestravaux/739 ; DOI : https://doi.org/ 10.4000/recherchestravaux.739 
Marie Bernanoce

Université Grenoble Alpes

UR LITT\&ARTS

\section{Le couple générationnel dans le théâtre jeunesse : une dualité peut en cacher une autre}

L'une des idées reçues concernant les spécificités du théâtre en direction de la jeunesse concerne la nécessaire présence de héros enfants. Or, comme on peut le montrer facilement ${ }^{\mathrm{I}}$, beaucoup de ces pièces et spectacles ne s'appuient pas sur des personnages d'enfants. L'exemple le plus notable en est incontestablement l'œuvre fondatrice que fut Mamie Ouate en Papoâsie de Joël Jouanneau et Marie-Claire Le Pavec ${ }^{2}$, pièce créée en 1988 et mettant en jeu deux personnages adultes. Et l'on pourrait citer bien d'autres œuvres emblématiques d'une adresse aux jeunes excluant les identifications facilement réalistes, à commencer par Les trois petits vieux qui ne voulaient pas mourir de Suzanne van Lohuizen ${ }^{3}$ dont le titre est en soi éclairant.

Certes, le millier de pièces actuellement publiées en collections jeunesse, dans la sphère francophone, donne une réelle visibilité à des personnages d'enfants. En ce sens, ce théâtre joue un rôle important dans l'ensemble du théâtre, en appui sur ce qu’a pu observer Jean-Gabriel Nordmann : «Je trouve que l'enfant n'est pas assez présent comme individu, comme personne, dans le

I. M. Bernanoce, «Les relations adulte-enfant dans le répertoire de théâtre jeunesse, au-delà de l'identification miroir : de l'épique à l'éthique», dans A. Rezzouk, M. Tsimbidy (dir.), La Jeunesse au miroir. Les pouvoirs du personnage, Paris, L'Harmattan, 2012, p. 219-23I.

2. J. Jouanneau, M.-C. Le Pavec, Mamie Ouate en Papoâsie, comédie insulaire, Arles, Actes Sud-Papiers, 1989; Actes Sud-Papiers Heyoka jeunesse, 2000.

3. S. van Lohuizen., Les trois petits vieux qui ne voulaient pas mourir, Paris, L'Arche jeunesse, 2005 . 
théâtre en général ${ }^{4}$.» Cependant, à tenter de circonscrire le champ du théâtre jeunesse, c'est moins le personnage enfant que la relation entre adultes et enfants qui s'impose comme un élément constitutif de cette part du théâtre contemporain, ce que nous nommerons le couple générationnel.

La réflexion menée ici concernera ainsi les relations entre personnages enfants et personnages adultes voire âgés dans le répertoire de théâtre jeunesse, la question posée pouvant se formuler ainsi : le couple adulte/enfant ne cachet-il pas une dualité plus complexe, de nature philosophique? Pour répondre à cette interrogation, et après un survol des risques esthétiques et éthiques du couple adulte/enfant, nous explorerons les diverses formes que prend ce duo.

\section{Le couple générationnel : les risques d'un théâtre didactique}

Le stéréotype que nous avons évoqué correspond à un couple de personnages adulte/enfant se résumant à cette formule : c'est l'adulte qui sait et l'enfant doit apprendre de l'adulte. Conçu ainsi, le théâtre destiné à la jeunesse se présente comme un théâtre de formation plaçant la parole adulte en surplomb par rapport à l'enfant et faisant le pari d'une identification formatrice dont il est facile de percevoir les écueils moralisateurs.

Au-delà des seuls personnages, un tel engagement du théâtre à destination de la jeunesse a été fondateur. Il suffit de citer Léon Chancerels pour camper une époque : celle de l'avant et de l'après Seconde Guerre mondiale, quand des militants ont rêvé un théâtre pour et par les jeunes pour mieux changer l'école, l'homme et le monde. Ces relations entre théâtre et jeunesse ont durablement marqué la culture française, dans leurs liens au «théâtre élitaire pour tous» de Jean Vilar, et elles ont été revivifiées tout au long des trois décennies qui ont suivi mai 68. Nombreux sont les pionniers du théâtre pour les jeunes à avoir ainsi puisé à la double source contradictoire de ce que Bérénice Hamidi-Kim analyse d'une part comme un "théâtre ontologiquement politique» unanimiste et, d'autre part, un "théâtre révolutionnaire de combat»"

Parmi les pionniers du théâtre pour les jeunes, Maurice Yendt ${ }^{7}$ apparaît à ce titre tout à fait emblématique : s'il a construit sur des fondations clairement

4. M. Bernanoce, À la découverte de cent et une pièces. Répertoire critique du théâtre contemporain pour la jeunesse, Montreuil-Grenoble, Éditions Théâtrales - SCEREN-CRDP de Grenoble, 2006, p. 33I.

5. L. Chancerel, Le Théâtre et la jeunesse, Paris, Bourrelier et Cie, I94I.

6. B. Hamidi-Kim, Les Cités du «théâtre politique en France depuis 1989, Paris, L’Entretemps, 2013, p. 37.

7. Son œuvre dramatique comporte une douzaine de pièces publiées aux cahiers du soleil debout, éditions du TJA reprises pour partie par les éditions Lansman. Se reporter à l'ouvrage 
brechtiennes toute l'histoire du TJA (aujourd'hui TNG, Théâtre Nouvelle Génération), dans le même temps son essai Les Ravisseurs d'enfants, paru en 1989, revendiquait très clairement un théâtre d'art pour les enfants ${ }^{8}$, en dénonçant, dans le sillage des réflexions de Benjamin sur le théâtre prolétarien" les dérives d'un théâtre de propagande.

Les autres pionniers que furent Bruno Castan, Catherine Dasté, Richard Demarcy, Françoise Pillet, René Pillot se sont inscrits dans cette adresse complexe aux enfants et aux jeunes, en appui sur une foi dans les grands récits. Mais ce positionnement a aussi construit malgré lui les limites de son action dans le temps. D'une certaine manière, avec le rejet du brechtisme, avec la fin des grands récits et l'avènement du postmodernisme, le théâtre pour les jeunes s'est trouvé réduit à du sous-théâtre à visée didactique, au sens péjoratif du terme : de ce fait, la position d'autorité de l'adulte par rapport à l'enfant/ lecteur/spectateur s'est elle aussi retrouvée perçue de façon négative. De là vient sans aucun doute la difficulté de certains auteurs et metteurs en scène actuels à accepter que le théâtre pour les jeunes puisse avoir des spécificités, ce qui déjà habitait la réflexion de Catherine Dasté.

Même si elle a fortement régressé, cette veine fondatrice a enclenché une mécanique paradoxale. Voici la position amusée de Jean-Gabriel Nordman pour qui ce serait alors "comme si on voulait destiner Oh les beaux jours de Beckett à un public de troisième âge, Les Femmes savantes de Molière à un public féminin ou Les Nègres de Genêt à un public noir $[\ldots]^{10}{ }$. Cela a contribué à la marginalisation d'un théâtre jeune public véritablement professionnel : c'est ainsi que l'on a vu et que l'on voit encore, faute de formation, des comédiens surjouant l'infans, l'enfant qui ne sait pas.

Bien évidemment, les œuvres de cette période fondatrice qui sont restées n'ont pas nécessairement versé dans le didactisme. Le théâtre puissant de Bruno Castan en est un excellent exemple, qui a fortement contourné le modèle du couple initial adulte/enfant.

On comprend donc facilement que les enjeux de la période récente, depuis la dernière décennie du $\mathrm{xx}^{\mathrm{e}}$ siècle, ont consisté à dépasser les apories de ce didactisme. Plutôt que d'être conçue en termes linéaires, la relation de l'âge

suivant : C. Ailloud-Nicolas (dir.), Théatre contemporain et jeune public. Avec En Lettres rouges de Maurice Yendt, Lyon, CRDP de Lyon, 2003.

8. M. Yendt, Les Ravisseurs d'enfants, du théâtre et des jeunes spectateurs, Paris, Actes SudPapiers, 1989, p. 59.

9. W. Benjamin, "Programme pour un théâtre d'enfants prolétarien", Travail théâtral. Cahiers trimestriels, $\mathrm{n}^{\circ}$ 20, Lausanne, La Cité, 1975, p. 46-50.

Io. M. Bernanoce, À la découverte de cent et une pièces, ouvr. cité, p. 33 I. 
adulte à l'enfance cherche désormais à se penser selon des détours complexes qui contournent et démultiplient le modèle initial.

\section{Premier type de détournement : l'inversion du sens du surplomb}

Les effets du postmodernisme aidant, un renversement radical s'est opéré : dans un certain nombre de pièces de ces quinze dernières années, c'est l'enfant qui sait et l'adulte qui apprend de l'enfant. Cette autre figure du couple générationnel peut donner des formes un peu stéréotypées, mais cela nourrit aussi des dramaturgies défiant la mise en scène par l'indécidabilité de la fiction et le jeu sur le point de vue. Dans Petits Sauvages de David Almond" ${ }^{\text {II }}$ une mère, tout d'abord totalement déroutée par la folie qui saisit sa fille à la mort de son père, se met peu à peu à rentrer dans les visions de sa fille jusqu'à sembler les partager réellement. Karin Serres, quant à elle, use de l'animalité comme d'un révélateur de l'irrationalité adulte mal maîtrisée. C'est le cas de plusieurs de ses pièces et surtout de Louiselles ours: une jeune fille sans mère se sent suivie par un ours blanc en guise d'ange gardien, puis elle en voit peu à peu derrière chaque adulte. Son père, totalement désemparé et d'abord rétif, rentre finalement dans le monde de sa fille, sans que l'on sache si véritablement les ours blancs existent.

Dans la plupart de ses pièces, Suzanne Lebeau confronte la parole défaillante et mensongère des adultes, ainsi dénoncée, à la réalité vécue par les enfants et c'est cela même qui fonde son regard sur le monde. Cela donne une dramaturgie profondément épique, thématisant l'activité de la parole, nécessaire même si elle n'est pas toujours salvatrice. Dans Le Bruit des os qui craquent ${ }^{12}$, au récit de la fuite des deux enfants-soldats Elikia et Joseph, s'ajoutent les scènes de « comparution" d'Angélina, l'infirmière qui a accueilli les deux enfants à l'hôpital où ils se sont retrouvés au terme de leur fuite et où Elikia est morte. Lors de ses comparutions, Angelina devient porte-parole du récit qu'Elikia a transmis. Les effets nés de l'accumulation de couches de récit s'opposent clairement au silence des membres de la commission (que la structure dramaturgique de la pièce ne désigne pas comme étant le public). La parole vaut quand le silence est aveu de faiblesse. L'infans parle.

On peut analyser de la même façon l'ensemble du théâtre jeunesse de Joël Jouanneau où se construit et se radicalise un couple enfant abandonné/adulte absent-mort qui constitue l'un des fils conducteurs du genre auquel Jouanneau

II. D. Almond, Petits Sauvages, Arles, Heyoka jeunesse - Actes Sud-Papiers, 2008.

I2. S. Lebeau, Le Bruit des os qui craquent, Montreuil, Éditions Théâtrales, coll. "Théâtrales Jeunesse», 2008. 
donne une claire portée métathéâtrale, en particulier dans PinkPunK CirKus ${ }^{13}$ : la désertion de l'adulte construit en effet la source où l'enfant puise la force de sa créativité, et celle-ci est nourrie de la nécessité des mots avec lesquels il lui faut jouer sa vie. Il en est de même pour la plupart des pièces de Nathalie Papin. Si l'enfant peut avancer dans sa vie, c'est grâce à une quête existentielle dont les racines s'enfoncent dans l'incurie de l'adulte, mère dans Debout ${ }^{14}$, père dans Le Pays de rien ${ }^{15}$ et dans Un, deux, Rois ${ }^{16}$, dont la mère est absente. Et de nombreuses pièces racontent cette quête de l'enfant sans l'adulte ou contre l'adulte. Citons Bouh! de Mike Kenny ${ }^{17}$, traitant d'un sujet peu fréquent, l'autisme, figure radicale de l'opposition entre le monde adulte et le monde de l'enfant au regard de la parole. Or, Bouh et son frère Benny sont des enfants sans parents et "Le garçon" et "La fille» deviennent les spectateurs et les acteurs de leur vie. Là, le théâtre donne doublement la parole à l'enfant qui n’a pas réussi à la prendre.

Ces dramaturgies du couple générationnel, notons-le au passage, confèrent un autre relief à la thématique de la quête, trop souvent cantonnée à sa seule dimension formative et réaliste par l'identification de l'enfant au personnage de l'enfant : on peut en effet y voir aussi ce que j'ai analysé ailleurs ${ }^{18}$ comme $^{2}$ un "effet Petit Poucet» aux significations tout à la fois sociologiques et métaphysiques, et qui concerne autant les adultes que les enfants, perdus ensemble dans un monde sans certitudes, ployant sous les coups de boutoir de l'ogre économique et sociétal.

\section{Deuxième type de détournement : l'adulte et l'enfant sur un même pied}

Comme une réponse à la situation d'abandon de l'enfant, que partage aussi l'adulte, s'est ainsi construit dans le théâtre jeunesse un autre couple générationnel mettant l'adulte et l'enfant à hauteur égale, sans surplomb de l'un par rapport à l'autre.

L'enfant peut alors devenir une sorte de révélateur dans la vie de l'adulte, sur un plan à la fois métaphysique et dramaturgique. C'est le sens du couple générationnel dans le diptyque que constituent Le Pays de rien et Un, deux, Rois

I3. J. Jouanneau, PinkPunK CirKus, Arles, Heyoka jeunesse - Actes Sud-Papiers, 2011.

I4. N. Papin, Debout, Paris, L'École des Loisirs, coll. "Théâtre", 200I.

I5. N. Papin, Le Pays de rien, Paris, L'École des Loisirs, coll. «Théâtre», 2002.

I6. N. Papin, Un, Deux, Rois, Paris, L'École des Loisirs, coll. "Théâtre», 20 I2.

I7. M. Kenny, Bouh!, trad. S. Magois, Arles, Heyoka jeunesse - Actes Sud-Papiers, 2012.

I8. M. Bernanoce, Vers un théâtre contagieux, Montreuil, Éditions Théâtrales, coll. «Sur le théâtre», 20I2, p.25. 
de Nathalie Papin : dans les deux cas, la quête têtue de l'enfant oblige l'adulte à dépasser ses limites et c'est sur l'enfant que repose l'avancée de la pièce. Dans La Morsure de l'âne ${ }^{19}$, c'est la petite fille non encore née, Zoé, qui pousse son père virtuel, alors dans le coma, à faire le choix de la vie et à ne pas se laisser happer par la mort. Ce faisant, l'adulte rendra possible la naissance à l'enfant.

Dans Une chenille dans le cœur ${ }^{20}$ de Stéphane Jaubertie, s'entrelacent les itinéraires des deux personnages du Bûcheron et de L'Enfant : le premier est né en perdant sa mère, sous l'arbre auprès duquel il a construit sa cabane, la seconde serait née d'une conception merveilleuse entre une femme et un arbre devenu homme. L'Enfant a dû laisser sa mère, mourante, pour s'en aller chercher un corset de bois plus grand, chenille qui veut devenir papillon; le Bûcheron devra quitter l'ombre protectrice de son arbre et le couper pour faire vivre l'Enfant, au prix de sa mort qu'elle aura cependant apaisée.

Beaucoup d'autres pièces fonctionnent sur la base de cet enfant révélateur, parfois aussi réparateur comme c'est le cas dans Les ours dorment enfin de Geneviève Billette ${ }^{21}$. Un enfant vient se coller aux vitres de Sacha, qui se cache la vérité sur son désastre amoureux. La pièce se conclut sur une scène de souffle vital conjurant la double solitude de l'un et de l'autre.

La figure de l'enfant et du jeune, dans cette pièce comme dans les précédentes, s'offre au lecteur-spectateur avec une valeur salvatrice presque christique, non loin d'une forme de stéréotype que vient contrebalancer un ancrage fort dans la double adresse à l'enfant et à l'adulte. Ces dramaturgies vont ainsi conduire les adultes metteurs en scène et comédiens vers d'intéressants détours esthétiques semés d'embûches parmi lesquels la moindre n'est pas la présence d'une figure d'adulte intercesseur entre parole des adultes et parole des enfants.

\section{Troisième type de détournement : la ronde générationnelle}

Dans le prolongement des relais entre l'adulte et l'enfant que l'on vient de voir émerger, se construisent aussi de véritables bouclages métaphysiques et dramaturgiques par-delà les âges, placés sous le signe de la transmission.

Dans Costa le Rougee22, Sylvain Levey confronte trois générations. Le "papé» veut transmettre à son petit-fils Costa ce qui a fait la légende de sa vie passée de paysan puis de syndicaliste communiste. Entre eux deux, la génération du fils

19. N. Papin, La Morsure de l'âne, Paris, L'École des Loisirs, coll. "Théâtre», 2008.

20. S. Jaubertie, Une chenille dans le cœur, Montreuil, Éditions Théâtrales, coll. "Théâtrales Jeunesse», 2008.

2I. G. Billette, Les ours dorment enfin, Carnières-Morlanwelz, Lansman, 2010.

22. S. Levey, Costa le Rouge, Montreuil, Éditions Théâtrales, coll. "Théâtrales Jeunesse», 20 II. 
de quarante ans, «Pa», qui a abandonné la lutte. Entre intime et politique, un homme de quatre-vingts ans doit accepter de mourir; un fils doit apprendre à son propre fils que son grand-père va mourir et il n'y parvient pas, et l'enfant, en passant par-dessus son père, prend alors le relais de la révolte dont la vie de son grand-père s'est nourrie.

Sur des thématiques intimes proches, Le Jardinier de Mike Kenny ${ }^{23}$ se nourrit des liens transgénérationnels dans un subtil mélange épique/dramatique. Dans la première des quatre parties de cette pièce intitulée "printemps", Joe raconte comment il s'est senti rejeté par ses parents à la naissance de sa petite sœur Florence dont il a enterré la poupée sous les yeux de son grandoncle, alors âgé et touché par la maladie d'Alzheimer. Joe enfant raconte alors les raisons de son geste à "L'Oncle Harry» dont la sœur, elle aussi nommée Florence, est sa grand-mère. C'est en "hiver» que Joe revoit Harry pour la dernière fois. Il déterre la poupée de sa sœur et la lui redonne. L'épilogue, intitulé "retour au présent", confirme les ressemblances entre Harry et Joe. Joe lui aussi a des troubles de mémoire et il est devenu jardinier. La transmission s'est accomplie. Notons au passage la place du regard dans la dramaturgie de cette pièce, avec ses nombreux "Il me regarde Je le regarde.» Nous sommes bien dans des jeux de miroirs et de reflets.

Cela se retrouve de façon forte dans L'Oubliance, que Mike Kenny présente comme «une image en miroir ${ }^{24}$ » de sa pièce Le Jardinier. Un vieil homme, Tobias, visiblement atteint de la maladie d'Alzheimer, se croit encore enfant alors qu'il vient d'arriver dans une maison de retraite où le personnel le traite précisément comme un enfant. Les autres vieillards commencent par se moquer de lui, non sans une certaine cruauté, puis ils finissent par le suivre dans sa douce folie de retour à l'enfance. Quand sa fille vient lui rendre visite, elle est accompagnée de son arrière-petit-fils nommé Toby. La ronde générationnelle est donc en place et le dénouement va donner plus de vigueur encore à cette force de vie que défend la dualité des prénoms Tobias/Toby. Mais le plus étonnant est peut-être la note didascalique qui suit la liste de personnages : "La convention, dans cette pièce, est que les jeunes acteurs habitent des corps âgés. Plus l'acteur sera jeune, plus le personnage qu'il jouera sera vieux. Et inversement ${ }^{25}$."

Cette ronde générationnelle rejoint le cas de toutes les pièces confrontant un enfant à la mort du vieillard qu'il faut apprivoiser, après l'avoir farouchement

23. M. Kenny, Le Jardinier, Arles, Heyoka jeunesse - Actes Sud-Papiers 2006.

24. M. Kenny, L'Oubliance, dans Si j’étais grand?, Montreuil, Éditions Théâtrales, coll. "Théâtrales Jeunesse», 2010.

25. Ibid., p. I2. 
refusée : c'est ainsi le cas dans Pacamambo de Wajdi Mouawad ${ }^{26}$ ou encore dans La Nuit MêmePasPeur de Claudine Galea ${ }^{27}$.

On peut aussi trouver, mais beaucoup moins fréquente, une figure inverse du couple précédent, la mort de l'enfant laissant des adultes abandonnés. Dans Azur d'Emma Hachée ${ }^{28}$, un petit garçon est en train de mourir et regarde de loin des parents qu'il va quitter. Quelques autres pièces jeunesse se confrontent aussi au personnage de l'enfant mourant, ainsi Mathieu trop court, François trop long de Jean-Rock Gaudreault ${ }^{29}$, mais on remarquera que l'adulte y est absent. La possible identification du lecteur-spectateur enfant à un personnage d'enfant mourant, absente de l'ouvrage dirigé par Georges Banu, L'enfant qui meurt $^{30}$, porte une dimension imaginaire puissante, remontant aux sources de la mythologie. C'est tout le sens de la pièce jeunesse des Suédois Suzanne Osten et Pier Lysander, Médée $e^{31}$.

\section{Quatrième type de détournement : le double générationnel}

La thématique du double, à forte charge métathéâtrale, est assez présente dans le théâtre jeunesse : dans Catalina in fine, Fabrice Melquiot ${ }^{32}$ l'aborde de front en racontant l'histoire d'une jeune adolescente orpheline aux deux visages : quand l'un des deux rit, l'autre pleure. Confrontée au monde de l'usine, Catalina se prend d'amitié pour le vieil Honorin qu'elle rudoie un peu, mais pour mieux l'amener à rêver, colorer et honorer la vie, tandis qu'un personnage nommé «Moi» campe la figure de l'auteur.

On peut alors émettre l'hypothèse que l'ensemble du théâtre à destination des jeunes tire sa force d'une dualité profonde intrinsèque, qui est précisément celle du couple adulte/enfant que démultiplie la relation métaphysique à la vie et à la mort. C'est, me semble-t-il, ce que revendiquent clairement, dans leur structure dramaturgique, plusieurs pièces relevant de ce que je nomme le double générationnel, théâtralisant un couple générationnel gémellaire : deux personnages différents à un premier niveau de l'action, l'un vieux et l'autre

26. W. Mouawad, Pacamambo, Arles, Heyoka jeunesse - Actes Sud-Papiers, 2000.

27. C. Galea, La Nuit MêmePasPeur, Montpellier, Espaces 34 jeunesse, 2009.

28. E. Haché, Azur, Carnières-Morlanwelz, Lansman jeunesse, 2007.

29. J.-R. Gaudreault, Mathieu trop court, François trop long, Carnières-Morlanwelz, Lansman, 1997.

30. G. Banu (éd., avec la collaboration de I. Ansart et V. Perruchon), L'Enfant qui meurt, Motif avec variations, Paris, l'Entretemps, 2OII.

3I. S. Osten, P. Lysander, Les Enfants de Médée, trad. du suédois M. Segol-Samoy, Montreuil, Éditions Théâtrales, coll. "Théâtrales Jeunesse», 2009.

32. F. Melquiot, Catalina in fine, Paris, L'Arche jeunesse, 2005. 
enfant, se révèlent être le même personnage à un second niveau, enchâssant, dans une forme particulière de monodrame qui défie la mise en scène de belle façon.

Si l'on reprend le fil du théâtre de Fabrice Melquiot, on découvre une pièce d'une grande richesse, Blanches ${ }^{33}$. Ouais a dix ans, elle passe beaucoup de temps avec sa grand-mère, Mémé Blanche, une vielle dame quelque peu excentrique qui a perdu son mari trois mois plus tôt et qui commence à ressentir les effets de la maladie d'Alzheimer. Dans la séquence vingt-six, elle est placée en maison spécialisée. Dans la dernière séquence, le dialogue entre elle et sa petite fille dépasse les frontières de la mort et Ouais reçoit enfin son vrai prénom, identique à celui de sa grand-mère décédée. Il y a donc deux Blanches dans cette histoire, d'où le pluriel du titre. Mais des indices se glissent ici et là dans la pièce jusqu'à l'ouvrir au double générationnel. Blanche âgée et Blanche enfant ne seraient-elles pas deux faces du même personnage? Blanches peut alors se lire aussi comme un monodrame, l'action se passant à l'intérieur de Blanche morte, dialoguant avec elle-même en se rejouant son enfance ou en se la réinventant, sans que cela soit exclusif d'autres lectures.

Le procédé est le même dans la pièce de Joël Jouanneau Dernier Rayon ${ }^{34}$. Il s'agit en effet au premier niveau de la dernière course d'un vieil homme, Aldébaran, avec un jeune garçon qu'il initie au vélo mais toute la pièce fonctionne sur une dramaturgie monodramatique, comme une scène intérieure. À un grand nombre de détails, on devine en effet que l'enfant n'est autre qu'un double d'Aldébaran au début de sa vie et que le vieil homme, avant de mourir, part à la recherche de son enfance.

Le sens philosophique du procédé se trouve exprimé par Philippe Dorin dans l'exergue de sa pièce Dans ma maison de papier, j'ai des poèmes sur le feu:

Tous les enfants

Sont à l'intérieur d'une vieille personne,

Mais ils ne le savent pas encore ${ }^{35}$.

Entre les deux personnages de La Vieille Dame et de la Petite Fille, le noir et la lumière, ainsi qu'un personnage-relais énigmatique, Le Promeneur, dans lequel on peut voir la figure du voyage intérieur d'un âge à l'autre autant que celle de l'auteur, comme dans Catalina in fine.

33. F. Melquiot, Blanches, Paris, L'Arche jeunesse, 20 Io.

34. J. Jouanneau, Dernier Rayon, Paris, L'École des Loisirs, coll. "Théâtre», I998.

35. P. Dorin, Dans ma maison de papier, j’ai des poèmes sur le feu, Paris, L'École des Loisirs, coll. «Théâtre», 2002, p. 9. 


\section{Du couple au double générationnel : une affaire de "blocs d'enfance»}

Le dialogue entre les âges, du couple au double générationnel, semble bel et bien définir l'adresse aux jeunes, consciente ou inconsciente. Écrire, lire et mettre en scène du théâtre en direction de la jeunesse n'est pas s'appuyer sur des souvenirs d'enfance. Bachelard dans Poétique de la rêverie évoque des "noyaux d'enfance». Deleuze quant à lui détecte chez Kafka des «blocs d'enfance»:

Le souvenir d'enfance est incurablement œedipien [...] le bloc d'enfance fonctionne tout autrement : il est la seule vraie vie de l'enfant; il est déterritorialisant; il se déplace dans le temps, avec le temps, pour réactiver le désir et en faire proliférer les connexions; il est intensif et, même dans le plus basses intensivités, en relance une haute ${ }^{36}$.

Le théâtre en direction de la jeunesse construit un théâtre mineur, au sens deleuzien, qui démultiplie la théâtralité : écrire et/ou publier du théâtre jeunesse, c'est mettre en dialogue différents âges de la vie et regarder le monde du point de vue de l'enfance, fictionnalisé en soi. Lire du théâtre jeunesse, c'est se couler dans ce double point de vue et le recontextualiser. C'est en cela que cette part du théâtre s'adresse autant aux adultes qu'aux enfants. Le rapport à l'enfance, dans sa naïveté feinte, interroge le monde par un détour dont adultes et enfants peuvent partager la nécessité. Les meilleures pièces jeunesse ne sont pas celles qui savent mériter aussi les adultes. Les meilleures pièces jeunesse sont celles qui construisent un mode de détour et de déterritorialisation pour dire le monde suffisamment riche et complexe pour que chacun ait la liberté de recontextualiser à sa façon le point de vue dynamique endossé, dans le dialogue entre les âges de la vie. Le dédoublement des âges peut alors se révéler fondateur d'une profonde exigence esthétique et éthique.

36. G. Deleuze, F. Guattari, Mille Plateaux, Paris, Minuit, 1980, p. 360. 